\title{
Histological and immunohistochemical characterisation of uterine adenocarcinoma in an Asian elephant (Elephas Maximus)
}

\author{
P. Laricchiuta1', V. Russo², A. Costagliola², G. Piegari², M. Capasso¹, P. Silvestre', \\ M. Martano², O. Paciello² \\ 'Zoological Garden Lo Zoo di Napoli, Naples, Italy \\ 2Department of Veterinary Medicine and Animal Production, University of Naples Federico II, Naples, Italy
}

[Received: 31 October 2017; Accepted: 28 January 2018]

\begin{abstract}
A 56-year-old nulliparous female Asian elephant (Elephas maximus) living at the zoological garden of Naples (Italy), with a clinical history of recurrent colic, was found in agonal state and humane euthanasia was elected. At necropsy the uterine body was moderately increased in size and the lumen was reduced due to a poorly demarcated and infiltrative neoplasm. Furthermore, multiple, whitish, firm nodules were present in both lungs. Histological examination of the uterine mass revealed epithelial cells arranged in tubular or solid pattern infiltrating the endometrium and the muscular layer. Immunohistochemical examination showed immunoreactivity of neoplastic cells to oestrogen receptors antibody. Pulmonary lesions were histologically and immunohistochemically superimposable to the epithelial uterine neoplasm. A definitive diagnosis of uterine adenocarcinoma with pulmonary metastases was made. (Folia Morphol 2018; 77, 4: 771-774)
\end{abstract}

Key words: uterine adenocarcinoma, elephant, elephas maximus, uterus

\section{INTRODUCTION}

Endometrial cancer is the most common women pelvic malignancy [2], and is rare in domestic animals other than rabbits $[3,11]$, cattle and Wistar rats [3]; it is exceptionally reported in other species, such as cats and dogs $[9,15]$. Cases of uterine adenocarcinoma are also described in rhinoceroses $[10,18]$ and many captive and wild animals $[12,16]$. In the female genital system of the elephant, several cases of leiomyomas are described $[5,14]$ but, to our knowledge, no cases of uterine adenocarcinoma have been ever diagnosed. The present study reports the first case of uterine adenocarcinoma in an Elephas maximus.

\section{CASE REPORT}

A 56-year-old nulliparous female Asian elephant (Elephas maximus) living at the zoological garden of
Naples (Italy), with a clinical history of recurrent colic, was found in lateral recumbency (agonal state) and given the lack of response to the emergency medical treatments, humane euthanasia was elected and a complete necropsy was requested to find out the cause of the illness of the animal.

At necropsy the uterine body was moderately increased in size and firmer than normal. The lumen was reduced due to a poorly demarcated and infiltrative neoplasm showing papillary and soft luminal projections and multiple brownish necrotic areas. Furthermore, multiple, disseminated, whitish to grey, well demarcated neoplastic nodules, ranging from 2 to $5 \mathrm{~cm}$ in diameter, involving both uterine horns and the uterine body, were also observed. Multiple, whitish, firm nodules, 1 to $4 \mathrm{~cm}$ in size were present in the lungs. Bulging into the right cardiac ventricle, and

Address for correspondence: O. Paciello, DVM, PhD, MSc., Department of Veterinary Medicine and Animal Production, Unit of Pathology, University of Naples Federico II, Via F. Delpino 1, 80137 Naples, Italy, tel: +39 081-2536466, e-mail: paciello@unina.it 


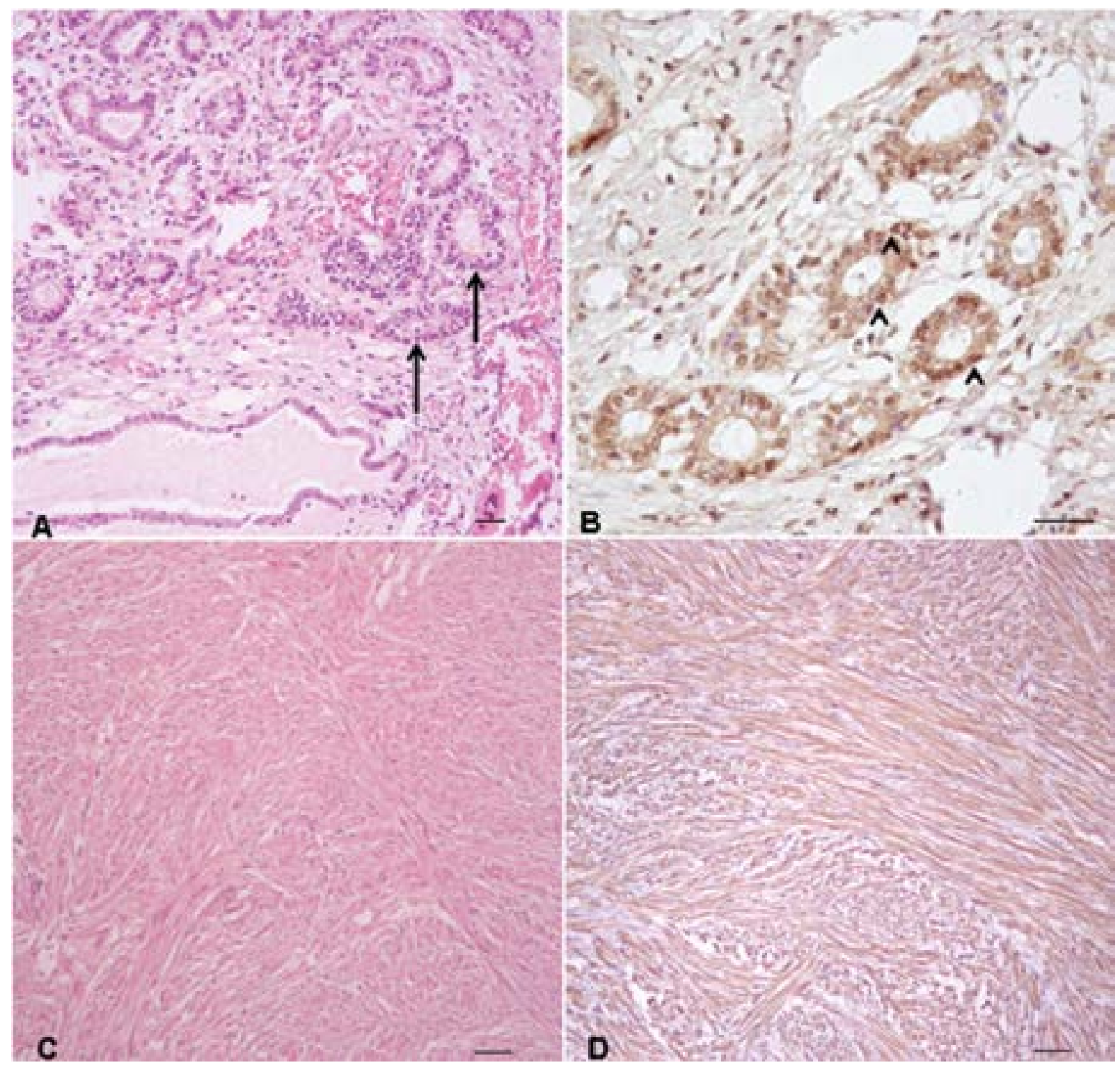

Figure 1. Microscopic images of uterine adenocarcinoma and leiomyoma in an Asian elephant (Elephas maximus); A. Uterine adenocarcinoma; neoplastic cells appear organised in acinar and tubular structures (arrows), with polygonal shape, distinct cell borders and eosinophilic to light basophilic cytoplasm; haematoxylin and eosin (H\&E) stain; B. Uterine adenocarcinoma; neoplastic cells show a strong nuclear immunoreactivity (arrow-head) to oestrogen receptor antibody; immunohistochemistry (IHC) stain; C. Uterine leyomioma; the cells are arranged in interlacing bundles or whorls and are supported by a moderate amount of fibrovascular stroma; H\&E stain; D. Uterine leyomioma: neoplastic cells showing strong immunoreaction to smooth muscle actin antibody; IHC stain; scale-bar 100 microns.

adhering to the endocardium, a firm to elastic reddish thrombus, $3 \mathrm{~cm}$ in diameter, was present. Based on the macroscopic examination, a suspected diagnosis of uterine adenocarcinoma with pulmonary metastasis and multiple uterine leiomyomas was made.

Representative samples of all organs were collected for a full histopathologic examination; tissues were fixed in $10 \%$ neutral buffered formalin, paraffin embedded, sectioned at 4 micron and stained with haematoxylin and eosin (H\&E). Histologically, the poorly demarcated and infiltrative neoplasm of the uterus consisted of an infiltrative unencapsulated neoplastic proliferation composed of epithelial cells infiltrating the endometrium and the muscular layer. Neoplastic cells formed acinar and tubular structures or solid aggregates and were polygonal in shape, with distinct cell borders and moderate eosinophilic to light basophilic cytoplasm (Fig 1A). Nuclei were oval with marginated or granular chromatin with a single prominent nucleolus; cellular atypia consisted of marked anisokaryosis and anisocytosis and mitotic figures ranged from 2 to 4 per high power field 
(HPF, 400 $\times$ magnification). Multifocal aggregates of neoplastic cells were noted in myometrial lymphatic vessels. On histological examination a diagnosis of uterine adenocarcinoma was made and immunohistochemistry (IHC) for pancytokeratin, oestrogen receptors (ER) and progesterone receptors (PR) was performed. Neoplastic cells showed strong cytoplasmatic immunoreaction to pancytokeratin antibody; the nuclei of tumour cells were intensely and diffusely labelled by marker for ER (Fig. 1B) whereas no expression of PR was found.

Furthermore, multiple, well-demarcated nodular uterine masses consisted of a relatively uniform population of mesenchymal cells arranged in interlacing bundles or whorls and supported by a moderate amount of fibrovascular stroma, were observed (Fig. 1C). The neoplastic cells were spindloid, often showed cigar-shaped nuclei and a mild to moderate anisokaryosis and anisocytosis; mitotic figures were rare. At IHC, neoplastic cells showed strong immunoreaction to smooth muscle actin antibody (Fig. 1D).

Pulmonary lesions were histologically and immunohistochemically superimposable to the epithelial uterine neoplasm (Fig. 2). Aggregates of neoplastic cells were found in mediastinal lymph nodes. On histological and IHC data a definitive diagnosis of uterine adenocarcinoma with pulmonary metastases and multiple uterine leiomyomas was made.

\section{DISCUSSION}

The classification of the uterus tumours of the domestic animals follows the general scheme employed in the first International Histological Classification of Tumours of Domestic Animals based on histological criteria. The classification includes: epithelial tumours such as adenoma and adenocarcinoma of the endometrium and mesenchymal tumours that include fibroma, leiomyoma and leiomyosarcoma [4].

Histological and immunohistochemical characteristics of multiple uterine leiomyomas have already been described in domesticated Asian elephant, and our findings are similar to those previously reported [5]; the tumourigenesis of uterine leiomyoma remains unclear even if previous studies have pointed out that hyperoestrogenic state is relevant to the initiation of this tumour [5].

To the author knowledge this is the first report of uterine adenocarcinoma with distant metastases diagnosed in an Asian elephant (Elephas maximus). In contrast, endometrial carcinoma is the most common

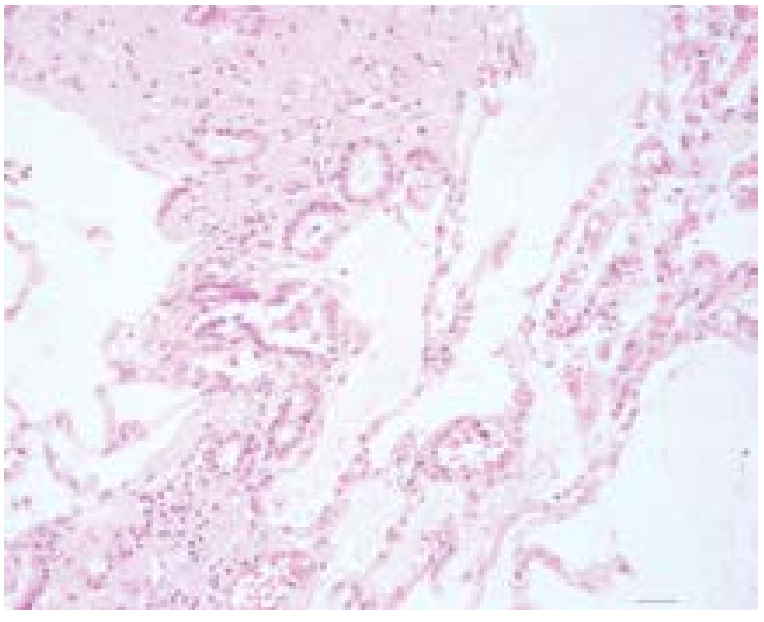

Figure 2. Lung; neoplastic cells appear organised in acinar and tubular structures, with polygonal shape, distinct cell borders and eosinophilic to light basophilic cytoplasm; haematoxylin and eosin stain; scale-bar 100 microns.

invasive neoplasm of the human female reproductive tract [2]. Chronic estrogenic stimulation has been associated with endometrial hyperplasia and carcinoma in women, and endometrial carcinoma tends to be most common in aging, nulliparous females with a history of menstrual irregularity consistent with anovulatory cycling [6]. This relation between sex hormones, especially oestrogens, and endometrial carcinoma is also described in rabbit [12].

To describe a case of uterus metastatic adenocarcinoma associated to multiple leiomyomas is extremely important also for epidemiological purpose considered that the elephants, despite their long life-span, seem to be at lower risk to develop cancer potentially related to multiple copies of TP53 gene. TP53 (encoding the protein p53 [RefSeq NM_000546]) is a crucial tumour suppressor gene, mutated in the majority of human and animal cancers [7]. Compared with human cells, elephant cells demonstrated increased apoptotic response following DNA damage [1].

\section{CONCLUSIONS}

Finally, the tumours described in this study seems to have the same histological pattern and biological behaviour already described in women and domestic animals $[9,17]$. Considering that anthropogenic activities contribute to the development of neoplasia in wildlife species [8], and that endometrial cancer may be related to environmental pollutants [13] animals like elephants, living for long time in zoological gardens, could be considered sentinels of disturbed environments. 


\section{REFERENCES}

1. Abegglen LM, Caulin AF, Chan A, et al. Potential mechanisms for cancer resistance in elephants and comparative cellular response to DNA damage in humans. JAMA. 2015; 314(17): 1850-1860, doi: 10.1001/jama.2015.13134, indexed in Pubmed: 26447779.

2. Benshushan A. Endometrial adenocarcinoma in young patients: evaluation and fertility-preserving treatment. Eur J Obstet Gynecol Reprod Biol. 2004; 117(2): 132-137, doi: 10.1016/j.ejogrb.2004.05.015, indexed in Pubmed: 15541846.

3. Elsinghorst TA, Timmermans HJ, Hendriks HG. Comparative pathology of endometrial carcinoma. Vet Q. 1984; 6(4): 200-208, doi: 10.1080/01652176.1984.9693937, indexed in Pubmed: 6388139.

4. Kennedy PC, Cullen JM, Edwards JF. World health organization international histologic classification of tumors of domestic animals. Histological classification of tumors of the skin of domestic animals, 2 nd series, vol. III. Armed Forces Institute of Pathology, Washington. 1998.

5. Kidsadagon P, Hiroo M, Jiraporn S, et al. Histopathological and immunohistochemical characterization of spontaneous uterine leiomyomas in two captive asian elephants. Thai J Vet Med. 2015; 45(2): 289-294.

6. Kumar V, Abbas AK, Fausto N. Robbins and Cotran Pathologic basis of disease. In: Kumar V (ed). Elsevier, Philadelphia 2005: 1085-1088.

7. Marfe G, De Martino L, Tafani M, et al. A multicancer-like syndrome in a dog characterized by $\mathrm{p} 53$ and cell cycle-checkpoint kinase 2 (CHK2) mutations and sirtuin gene (SIRT1) down-regulation. Res Vet Sci. 2012; 93(1): 240-245, doi: 10.1016/j. rvsc.2011.07.030, indexed in Pubmed: 21890154.

8. McAloose D, Newton AL. Wildlife cancer: a conservation perspective. Nat Rev Cancer. 2009; 9(7): 517-526, doi: 10.1038/nrc2665, indexed in Pubmed: 19550426.
9. Meuten DJ. Tumors in Domestic Animals. In: Meuten DJ (ed). Wiley-Blackwel, lowa 2017: 557-558.

10. Panda SK, Nath I, Roy PK, et al. Uterine adenocarcinoma in an indian one-horned rhinoceros (rhinoceros unicornis). Zoos' Print. 2009; 24(7): 17-19.

11. Rahim A, Ahmad A, Reza K, et al. Uterine adenocarcinoma in a domestic rabbit. GV. 2012; 9(2): 245-247, doi: 10.5829/idosi.gv.2012.9.2.6513.

12. Robert N, Posthaus $\mathrm{H}$. Uterine adenocarcinoma in a captive sika deer. J Wildl Dis. 1999; 35(1): 141-144, doi: 10.7589/0090-3558-35.1.141, indexed in Pubmed: 10073366.

13. Rzymski P, Tomczyk K, Rzymski P, et al. Impact of heavy metals on the female reproductive system. Ann Agric Environ Med. 2015; 22(2): 259-264, doi: 10.5604/12321966.1152077, indexed in Pubmed: 26094520.

14. Sapundzhiev E, Pupaki D, Zahariev P, et al. Fibroleiomyoma in elephant uterus. J Vet Med A Physiol Pathol Clin Med. 2007; 54(9): 499-500, doi: 10.1111/j.14390442.2007.00949.x, indexed in Pubmed: 17931224.

15. Serenella $P$, Roperto F. Spontaneous Uterine Tumors in Three Cats. Vet Path. 1984; 21: 257-258.

16. Thompson R, Armién AG, Rasmussen JM, et al. Uterine adenocarcinoma in a Przewalski's wild horse (Equus ferus przewalskii). J Zoo Wildl Med. 2014; 45(2): 441-445, doi: 10.1638/2013-0200R1.1, indexed in Pubmed: 25000717.

17. Turan T, Ureyen I, Karalok A, et al. Pulmonary recurrence in patients with endometrial cancer. J Chin Med Assoc. 2016; 79(4): 212-220, doi: 10.1016/j.jcma.2015.10.010, indexed in Pubmed: 26874679.

18. Wilson M, Hermes R, Bainbridge J, et al. A case of metastatic uterine adenocarcinoma in a southern white rhinoceros (Ceratotherium simum simum). J Zoo Wildl Med. 2010; 41(1): 111-114, doi: 10.1638/2009-0128.1, indexed in Pubmed: 20722262. 